\title{
Mitochondrial Genome Sequences Effectively Reveal the Phylogeny of Hylobates Gibbons
}

\author{
Yi-Chiao Chan ${ }^{1 *}$, Christian Roos ${ }^{2}$, Miho Inoue-Murayama ${ }^{3}$, Eiji Inoue ${ }^{4}$, Chih-Chin Shih $^{5}$, Kurtis Jai-Chyi \\ Pei $^{6}$, Linda Vigilant ${ }^{1}$
}

1 Department of Primatology, Max-Planck Institute for Evolutionary Anthropology, Leipzig, Germany, 2 Gene Bank of Primates and Primate Genetics Laboratory, German Primate Center, Göttingen, Germany, 3 Wildlife Research Center, Kyoto University, Kyoto, Japan, 4 Graduate School of Science, Kyoto University, Kyoto, Japan, 5 Animal Division, Taipei Zoo, Taipei, Taiwan, 6 Institute of Wildlife Conservation, National Pingtung University of Science and Technology, Pingtung, Taiwan

\begin{abstract}
Background: Uniquely among hominoids, gibbons exist as multiple geographically contiguous taxa exhibiting distinctive behavioral, morphological, and karyotypic characteristics. However, our understanding of the evolutionary relationships of the various gibbons, especially among Hylobates species, is still limited because previous studies used limited taxon sampling or short mitochondrial DNA (mtDNA) sequences. Here we use mtDNA genome sequences to reconstruct gibbon phylogenetic relationships and reveal the pattern and timing of divergence events in gibbon evolutionary history.

Methodology/Principal Findings: We sequenced the mitochondrial genomes of 51 individuals representing 11 species belonging to three genera (Hylobates, Nomascus and Symphalangus) using the high-throughput 454 sequencing system with the parallel tagged sequencing approach. Three phylogenetic analyses (maximum likelihood, Bayesian analysis and neighbor-joining) depicted the gibbon phylogenetic relationships congruently and with strong support values. Most notably, we recover a well-supported phylogeny of the Hylobates gibbons. The estimation of divergence times using Bayesian analysis with relaxed clock model suggests a much more rapid speciation process in Hylobates than in Nomascus.

Conclusions/Significance: Use of more than $15 \mathrm{~kb}$ sequences of the mitochondrial genome provided more informative and robust data than previous studies of short mitochondrial segments (e.g., control region or cytochrome b) as shown by the reliable reconstruction of divergence patterns among Hylobates gibbons. Moreover, molecular dating of the mitogenomic divergence times implied that biogeographic change during the last five million years may be a factor promoting the speciation of Sundaland animals, including Hylobates species.
\end{abstract}

\footnotetext{
Citation: Chan Y-C, Roos C, Inoue-Murayama M, Inoue E, Shih C-C, et al. (2010) Mitochondrial Genome Sequences Effectively Reveal the Phylogeny of Hylobates Gibbons. PLoS ONE 5(12): e14419. doi:10.1371/journal.pone.0014419

Editor: Robert C. Fleischer, Smithsonian Institution National Zoological Park, United States of America

Received June 15, 2010; Accepted November 29, 2010; Published December 23, 2010

Copyright: (C) 2010 Chan et al. This is an open-access article distributed under the terms of the Creative Commons Attribution License, which permits unrestricted use, distribution, and reproduction in any medium, provided the original author and source are credited.

Funding: This work was supported by a grant from the Leakey Foundation (L.V.), the fellowship of the Deutscher Akademischer Austausch Dienst (Y.-C.C.) and the Max Planck Society. Leakey Foundation website: http://www.leakeyfoundation.org/. Deutscher Akademischer Austausch Dienst (DAAD) website: http://www. daad.de/en/index.html. Max Planck Society website: http://www.mpg.de/english/portal/index.html. The funders had no role in study design, data collection and analysis, decision to publish, or preparation of the manuscript.

Competing Interests: The authors have declared that no competing interests exist.

*E-mail: yichiao_chan@eva.mpg.de
}

\section{Introduction}

Gibbons (Hylobatidae) are small arboreal apes living in tropical and sub-tropical forests of the mainland and islands of Southeast Asia, including the Malay Peninsula, Sumatra, Borneo, Java and Mentawai Islands (Figure 1). Uniquely among contemporary hominoids, gibbons exist as multiple geographically contiguous taxon exhibiting distinctive behavioral, morphological, and karyotypic characteristics. Gibbons are classified into four genera Hylobates, Hoolock, Nomascus, and Symphalangus, each of which features a different number of chromosomes [1,2,3,4,5]. The number of species and subspecies is a more problematic issue, with some authorities listing as many as 28 potential taxa [2], while others limit themselves to 25 taxa and differ as to whether there may be 16 [5] or 18 species [4] therein. In contrast, two species of orangutans and gorillas have been recognized [1,2] along with the bonobo and four subspecies of chimpanzees [1] meaning that the speciosity of gibbons provides a startling contrast to the situation in other ape lineages.
The evolutionary relationships of gibbon taxa have long been a focus of study due to their high taxonomic diversity and conservation importance, as nearly all gibbons have been classified as endangered at either the species or subspecies level [5]. Numerous morphological and molecular studies on the phylogenetic relationships among Hylobatidae members have led to consensus on the monophyly of each of the four genera $[6,7,8,9,10]$. A scenario in which Nomascus diverged first, followed by Symphalangus, Hoolock and then Hylobates is well-supported by analyses of mtDNA control region segments [6], and is also inferred, albeit with weaker statistical support, in a more recent study of mtDNA cytochrome b (cyt b) sequences [4]. While the genera Hoolock and Symphalangus contain just two and one species, respectively, some half-dozen species have been attributed to both Nomascus and Hylobates. Recent analysis of mtDNA cyt b sequences produced a statistically supported description of phylogenetic relationships among Nomascus spp. [4,11]. In contrast, studies of short segments of mtDNA have proven unable to confidently resolve the phylogenetic relationships among the members of the 


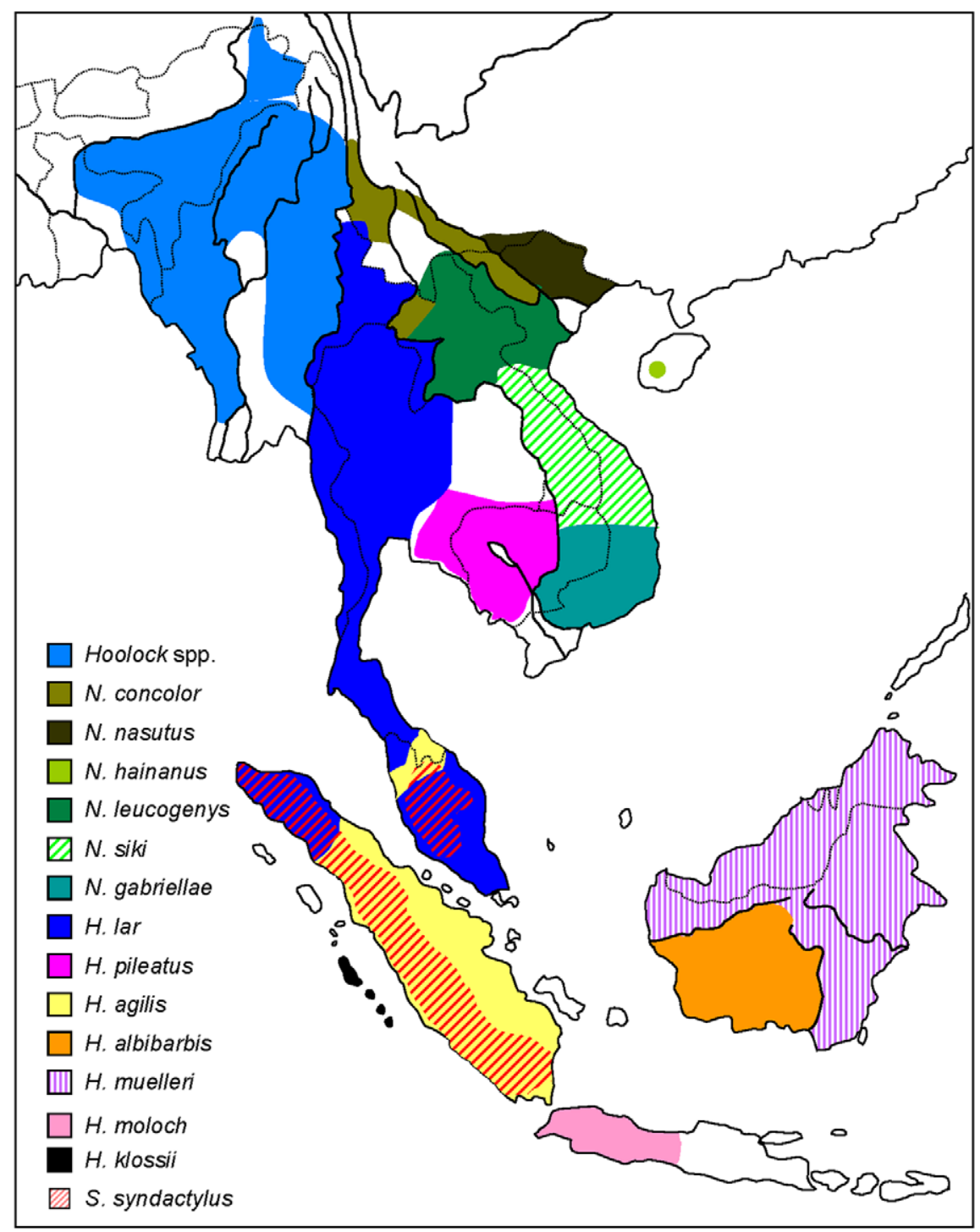

Figure 1. Approximate geographic distribution of gibbons. Dotted and solid lines indicate country borders and major rivers, respectively. Adapted from [4].

doi:10.1371/journal.pone.0014419.g001

genus Hylobates $[4,8,10,12,13]$. This suggests that while the evolutionary radiation of Nomascus gibbons appears to have occurred in a stepwise fashion over some 4 million years, in the case of Hylobates short mtDNA sequences (control region [10], cyt b [4,12], ND3, ND4 and ND5 genes [8,13] do not provide enough information for depicting their apparently rapid evolutionary radiation.

In addition to the pattern of evolutionary relationships, the timings of the molecular divergences within gibbons are also of interest. Results from mtDNA studies suggested that the split of great apes and gibbons occurred 15-20 million years ago (mya) $[4,14,15,16,17]$. Two studies relying on mtDNA cyt b sequences suggest that a rapid radiation of gibbons began some 8 to 10 mya $[4,18]$. In particular, the differentiation of the species within the Nomascus genera was inferred to have occurred from 4.2 mya to as recently as 0.55 mya [4]. It would be interesting to compare the timings of the divergences within Nomascus to those within the similarly speciose Hylobates genus, but this is hampered by the lack of a reliable phylogeny for Hylobates.
Recent phylogenetic studies reveal that longer mtDNA sequences, e.g. mitochondrial genomes (mtgenomes), can provide sufficient resolution for reconstructing a robust phylogeny $[19,20,21,22,23,24,25]$ and also facilitate the molecular dating of divergence events within a phylogeny $[16,17,22,23,24,25,26]$. Whole mtDNA genome sequences can be recovered efficiently by amplifying the entire $\sim 16 \mathrm{~kb}$ genome in one or two fragments, which also serves to reduce the chance of inadvertent analysis of segments of mtDNA that have translocated to the nuclear genome ('numts') [27]. Unfortunately, the amplification of such large segments of the mitochondrial genome demands high-quality DNA and is not successful when applied to the limited amount of degraded DNA obtained from non-invasively obtained materials such as hair or feces. Many gibbon taxa are present in very small populations and are not held in captivity, so that only non-invasive samples can be collected. For example, one recent study used DNAs from blood, tissues, feces and hair to achieve a very comprehensive sampling of gibbon taxa, but was necessarily limited to analysis of a small proportion of the mtDNA molecule 
and so not all inferences were supported with statistical confidence [4]. In contrast, researchers in another recent study generated whole mtDNA genome sequences from gibbons, but analyzed only five individuals representing three of the four genera, and thus although the topology was well-supported the insights were limited [15].

Here we sought to generate mitochondrial genome sequences from as many gibbons as possible in order to improve the resolution of the evolutionary relationships among members of the gibbon family and assess the timings of various divergence events. We generated DNA sequences of entire mitochondrial genomes from 51 gibbons representing three of the four genera and 11 different species. These data produce a reliable phylogeny featuring strong support values and most notably contribute to resolution of the phylogeny of Hylobates species and the timings of molecular divergence events.

\section{Results and Discussion}

\section{Gibbon mitochondrial genome sequences}

We produced whole mtDNA genome sequences from 51 individuals. From each individual we obtained an average of 1,965 tagged reads with an average length of $191 \mathrm{bp}$, thus yielding approximately $410 \mathrm{~kb}$ of sequence data corresponding to 24-fold coverage of the gibbon mitochondrial genome. We assembled tagged reads from each individual into consensus contigs within which sites with low coverage $(<5$-fold) and ambiguous sites were marked as missing data $(\mathrm{N})$. Within the 51 consensus contigs the percentage of missing data ranged between 0 and $13.54 \%$. The positions with missing data were further examined and found to mostly occur in the control region, so that for each individual the percentage of positions with missing data outside the control regions ranged from 0 to $8.84 \%$.

The 51 mtgenome sequences represent 11 gibbon species belonging to three genera, Hylobates, Nomascus and Symphalangus. All gibbon mitochondrial genomes consisted of the 22 tRNA genes, 2 rRNA genes, 13 protein-coding genes and the control region in the order typically observed in vertebrates. In common with other studies generating and analyzing whole mtDNA sequences for interspecific comparisons (e.g. [23]) we noted that the control region tends to contain sites with missing data and because of its high rate of evolution can be difficult to align among species. Therefore, we produced concatenated sequences of the 37 individual genes for further analyses. These concatenated sequences ranged from 15,407-15,416 bp in length.

\section{Phylogenetic analyses}

All three methods used for phylogeny reconstructions produced the same topology with strong support values (Figure 2). The three gibbon genera examined here appear as monophyletic clades with Nomascus diverging first followed by Symphalangus and Hylobates.

Within Nomascus, each of the four species represented here forms a well-supported monophyletic clade or, as in the case of $\mathcal{N}$. concolor, a distinct lineage. $\mathcal{N}$. concolor is the basal taxon here, but we lack samples from $\mathcal{N}$. hainanus and $\mathcal{N}$. nasutus which form the earliest divergence in other recent work [4]. Following the divergence of $\mathcal{N}$. concolor there is a divergence between $\mathcal{N}$. gabriellae and the $\mathcal{N}$. leucogenys $/ \mathcal{N}$. siki clade. $\mathcal{N}$. leucogenys and $\mathcal{N}$. siki are each monophyletic and exhibit a recent divergence from each other. These results are highly supported and entirely consistent with findings based upon analysis of the cyt b gene [4].

Our analysis of mtDNAs from six of seven species of Hylobates gibbons produces a well-supported phylogeny in which each species is represented by distinct lineages or monophyletic clades. The most closely related pairs of species consist of $H$. lar and $H$. pileatus, $H$. klossii and $H$. moloch, and $H$. agilis and $H$. muelleri. We lack samples from $H$. alibarbis, which would be expected to be closely related to $H$. agilis [4]. As is not unexpected, our Hylobates phylogeny differs from previous work that did not achieve statistical reliability $[4,8,10,12,13]$. These studies had relied on information from different mtDNA segments, e.g. control region $[10]$, cyt b $[4,12]$, ND3, ND4 and ND5 genes $[8,13]$. Use of only one of these segments provided too little information for confident resolution of a phylogeny featuring short branch lengths between divergences [8,10,22,28,29].

Unlike the other genera, the siamang (Symphalangus) only occurs as a single species, which is supported by the monophyly of the mtDNAs examined here.

\section{Estimation of divergence times}

Using three fossil calibration points we estimated mtDNA divergences times within gibbons (Table 1). The divergence time between gibbons and great apes was estimated at 19.25 million years ago. This is compatible with the Bayesian estimations of other mtgenomic studies $[15,16,17]$ and predates slightly the estimates of 16.26 mya based on the mtDNA cyt b gene [4].

Although we could not sample representatives of the genus Hoolock, our inference of the relationships among genera is otherwise similar to the well-supported phylogeny of genera presented by Roos and Geissmann [6], in which Hoolock diverges after Symphalangus but before Hylobates. Thus asssuming that the initial split within gibbons occurred between Nomascus and all remaining gibbons, we infer a date for this event of around 8.67 mya (for $95 \%$ confidence intervals see Table 1), in comparison with a recent estimate of 8.34 mya based on cyt b sequences [4]. The divergences among the four Nomascus species examined here began at around 2.4 mya. However, this is likely an underestimate as in comparison with the recent work by Thinh and coworkers we lack samples from $\mathcal{N}$. hainanus and $\mathcal{N}$. nasutus, which would be expected to form an early divergence in Nomascus that has been dated to 4.24 mya [4]. Our results concur in estimating the divergence of $\mathcal{N}$. gabriellae at 1.40 mya (1.74 mya in [4]). We find that the most recent species split of Nomascus gibbons occurred between $\mathcal{N}$. leucogenys and $\mathcal{N}$. siki at 0.46 mya, consistent with the date of 0.55 mya previously inferred [4]. In sum, divergence events within Nomascus appear to have occurred over a relatively long period of time, resulting in the appearance of some six species in more than 4 million years.

In contrast to the apparent stepwise fashion of divergences in Nomascus, the radiation of Hylobates gibbons began with an initial divergence at 4.17 mya, followed by a split between $H$. lar and $H$. pileatus at 2.90 mya and nearly contemporaneous molecular divergences between two additional species pairs (H. moloch and $H$. klossii, H. muelleri and $H$. agilis) at 2.77 and 2.62 mya, respectively. In contrast to the successive divergences of the Nomascus species, the species radiation within Hylobates occurred over a much shorter time period of only 1.5 million years, thus explaining the need for larger datasets to resolve the divergences within the genus with significance. Consistent with other studies, we infer that the radiation within Hylobates began at about 4 mya $[4,15]$.

There are three pairs of Hylobates species which come into contact and reportedly hybridize in sympatry. These species include $H$. albibarbis and $H$ muelleri, $H$ agilis and $H$. lar, and $H$. lar and $H$. pileatus [30]. In our study, all examined Hylobates species with multiple samples were monophyletic in the phylogenetic analyses, suggesting that no individuals were representative of 
Support values (ML/Bayesian/NJ)

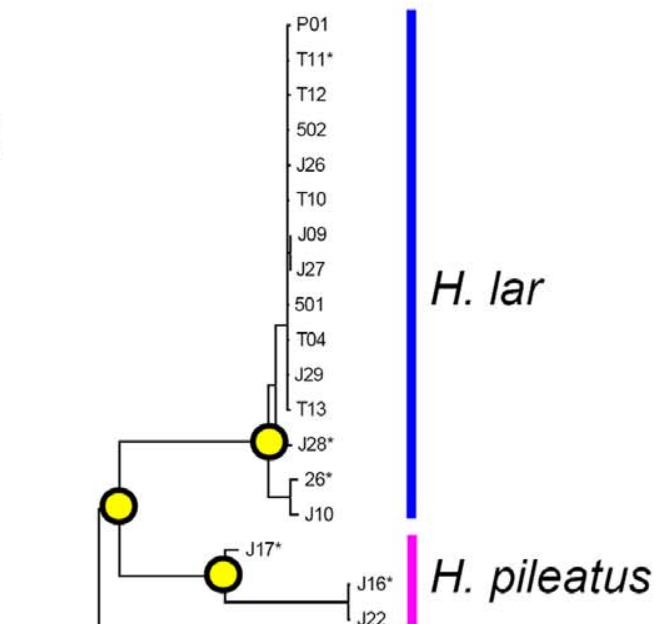

\section{Hylobates}

H. moloch

H. klossii

H. muelleri
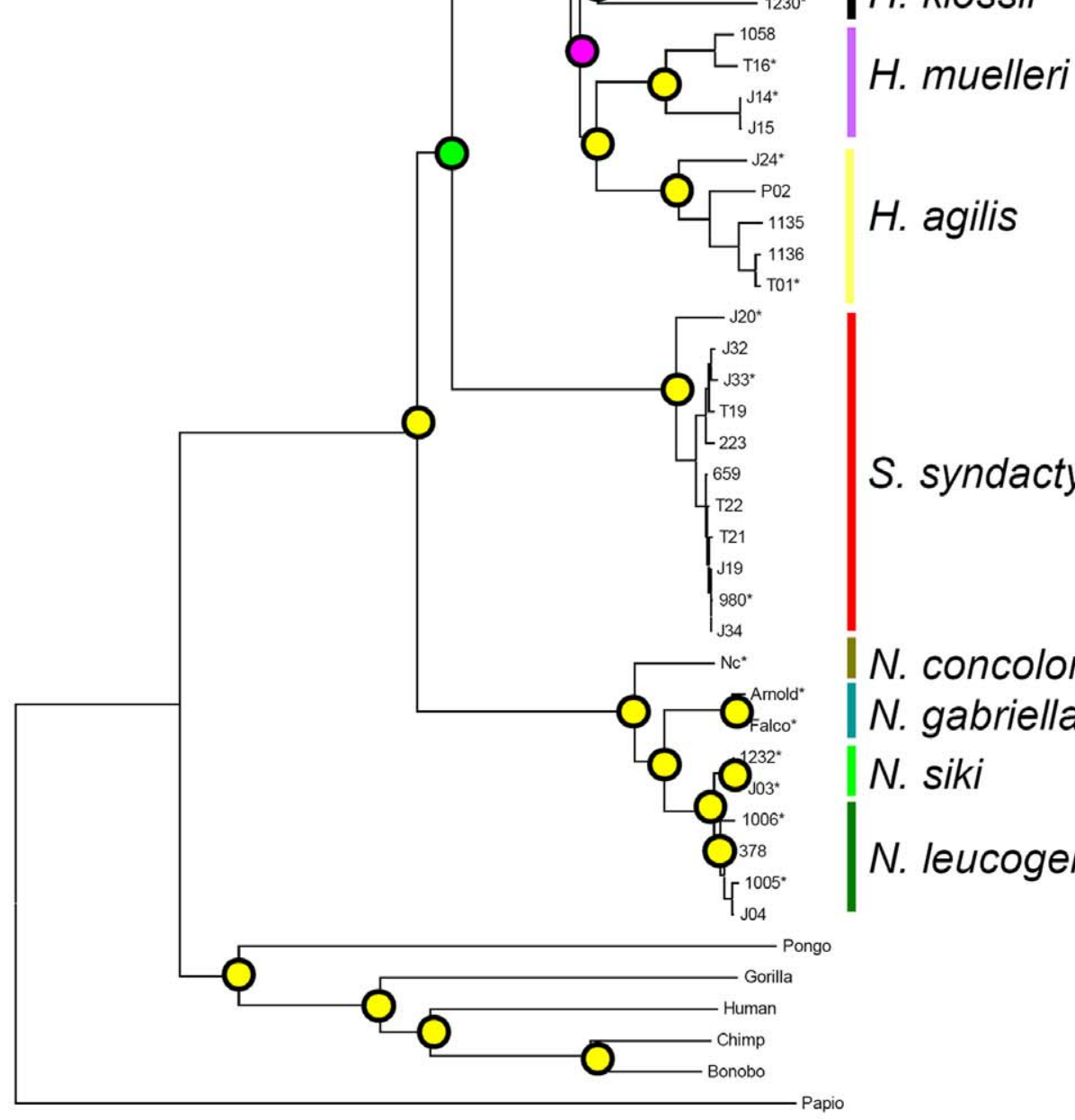

\section{$H$. agilis}

S. syndactylus

Symphalangus

N. concolor

N. gabriellae

N. siki

N. leucogenys
Nomascus

Figure 2. Phylogenetic tree of gibbons and outgroup primates based on the mtDNA concatenated dataset. The phylogenetic relationships among gibbons and six outgroup primates were inferred from the mtDNA concatenated dataset, including three partitioned sets: ribosomal RNA, transfer RNA and protein-coding gene. The maximum likelihood (ML), Bayesian and neighbor-joining (NJ) methods were used to reconstruct phylogenetic trees. All three analyses produced the same topology and their support values are indicated by circles on the nodes of the $\mathrm{NJ}$ tree shown here. Individuals used in the estimation of divergence times are marked with an asterisk. doi:10.1371/journal.pone.0014419.g002 
Table 1. Bayesian estimates of divergence times based on the relaxed molecular clock approach.

\begin{tabular}{|c|c|c|c|}
\hline Node & Divergence & mean & 95\% HPD \\
\hline $\mathrm{t} 1^{*}$ & Baboon (Papio)-apes & 22.96 & $21.01-24.82$ \\
\hline t2 & Great apes-Gibbons & 19.25 & $15.54-22.99$ \\
\hline t3 & Gibbons (Nomascus-other gibbon spp.) & 8.67 & $5.33-12.53$ \\
\hline t4 & Symphalangus-Hylobates & 7.52 & $4.48-10.94$ \\
\hline t5 & Hylobates (2 Hylobates subclades) & 4.17 & $2.48-6.13$ \\
\hline t6 & H. lar-H. pileatus & 2.90 & $1.45-4.5$ \\
\hline t7 & 2 Hylobates sister groups (H. moloch/H.klossii-H. muelleri/H. agilis) & 3.45 & $2.04-5.1$ \\
\hline t8 & H. moloch-H. klossii & 2.77 & $1.47-4.19$ \\
\hline t9 & H. muelleri-H. agilis & 2.62 & $1.47-4$ \\
\hline $\mathrm{t} 10$ & N. concolor-other Nomascus spp. & 2.37 & $1.07-4$ \\
\hline $\mathrm{t} 11$ & N. gabriellae- N. leucogenys/N. siki & 1.40 & $0.57-2.44$ \\
\hline $\mathrm{t} 12$ & N. leucogenys-N. siki & 0.46 & $0.19-0.82$ \\
\hline $\mathrm{t} 13$ & MRCA Symphalangus & 0.81 & $0.31-1.53$ \\
\hline $\mathrm{t} 14$ & MRCA H. lar & 0.50 & $0.19-0.91$ \\
\hline $\mathrm{t} 15$ & MRCA H. pileatus & 0.71 & $0.35-1.15$ \\
\hline $\mathrm{t} 16$ & MRCA H. moloch & 0.40 & $0.11-0.79$ \\
\hline $\mathrm{t} 17$ & MRCA H. muelleri & 1.24 & $0.47-2.19$ \\
\hline $\mathrm{t} 18$ & MRCA H. agilis & 0.92 & $0.36-1.66$ \\
\hline t19 & MRCA N. gabriellae & 0.21 & $0.06-0.41$ \\
\hline $\mathrm{t} 20$ & MRCA N. siki & 0.06 & $0.01-0.12$ \\
\hline $\mathrm{t} 21$ & MRCA N. leucogenys & 0.26 & $0.08-0.49$ \\
\hline $\mathrm{t} 22^{*}$ & Great apes (orangutan-other great apes) & 14.02 & $12.24-15.89$ \\
\hline $\mathrm{t} 23$ & Gorilla-Human/Pan & 8.95 & $6.95-11.08$ \\
\hline $\mathrm{t} 24^{*}$ & Human-Pan & 6.35 & $5.41-7.26$ \\
\hline $\mathrm{t} 25$ & Pan (chimpanzee-bonobo) & 2.38 & $0.94-4.04$ \\
\hline
\end{tabular}

The divergence time estimates are in million years before present. MRCA denotes the most recent common ancestor. HPD, highest posterior density.

*Nodes used for calibration.

doi:10.1371/journal.pone.0014419.t001

recent female-mediated gene flow between species. Studies of multiple independent genetic markers, ideally using samples of known geographic provenance, would be needed to effectively address the question of the extent and consequences of gene flow among Hylobates species.

\section{Broader conclusions}

Using mtDNA genome sequences to analyze the phylogenetic relationships of 11 extant gibbon species belonging to three genera, we obtained a phylogeny characterized by confident support values of all nodes and most noteworthily, among Hylobates species. We showed that complete mtgenome sequences provide sufficient information to resolve the sequence of events in the rapid divergence of Hylobates. The Bayesian inferences of split times were consistent with previous work. However, it is interesting to note that the confidence intervals surrounding those divergence time estimates are still rather large (Table 1). Although our divergence time estimates are generally similar to those obtained by other researchers using smaller mtDNA segments (e.g. cyt b [4]), the confidence intervals do not appear to have narrowed despite the use of this larger dataset. For example, the width of the confidence interval around the estimated 19.25 mya great ape and gibbon divergence is $7.45 \mathrm{my}$, even larger than the $3.47 \mathrm{my}$ interval around the estimate of 16.26 mya previously estimated [4]. Since we used the same fossil-based calibration points as previous researchers and identical settings for the analysis, this result is most likely attributable to the difficulties inherent in modeling the inherently heterogeneous patterns of mutation of various mtDNA protein-coding genes.

We estimated that the timings of most splitting events in the Hylobatidae family occurred from the late Miocene to the Pliocene (11.6-2.6 mya), as was also observed in other diverse mammalian families, including bears (the Ursidae, [21]), modern cats (the Felidae, [31]), deer (the Cervidae, [32]), and true seals (the Phocidae, [33]). The coincidence of speciation times across different families implied that environmental changes around that time may have played an essential role in the evolutionary processes of gibbons and other mammals $[34,35,36]$. Of all gibbon genera, Hylobates has the largest distribution spreading throughout the Sundaland, which is characterized by high levels of species richness and endemism (biodiversity hotspot, [37]). The divergence events within the Hylobates phylogeny were estimated to have occurred in the Pliocene (5.3-2.6 mya). During this period, speciation events were also reported for other Sundaland animals, e.g. mutualistic Crematogaster ants [38], Ficedula flycatchers [39] and Sundasciurus tree squirrels [40]. We suggest that biogeographic changes in Southeast Asia in the past five million years, particularly those induced by sea level changes [41], may have been a factor promoting the evolutionary divergence of terrestrial animals in the Sundaland. 
Table 2. Gibbon samples used in the present study.

\begin{tabular}{|c|c|c|c|c|c|}
\hline Genus & Species & onwer's ID/Barcode ID & working ID & origin $^{a}$ & current deposition $^{a}$ \\
\hline \multirow[t]{31}{*}{ Hylobates } & agilis & $20050292 \mathrm{D} 10$ & T01 & Taipei Zoo & Taipei Zoo \\
\hline & & 1135 & 1135 & Bristol Zoo & German Primate Center \\
\hline & & 1136 & 1136 & Bristol Zoo & German Primate Center \\
\hline & & $941006 \mathrm{G} 01$ & P02 & Pingtung Rescue Center & Pingtung Rescue Center \\
\hline & & 1061 & $J 24$ & Ragunan Zoo & WRC, Kyoto University \\
\hline & lar & 20040082D10 & T04 & Taipei Zoo & Taipei Zoo \\
\hline & & 20040113D10 & T10 & Taipei Zoo & Taipei Zoo \\
\hline & & $20040284 \mathrm{D} 10$ & T11 & Taipei Zoo & Taipei Zoo \\
\hline & & 20040285D10 & T12 & Taipei Zoo & Taipei Zoo \\
\hline & & $20040286 \mathrm{D} 10$ & $\mathrm{~T} 13$ & Taipei Zoo & Taipei Zoo \\
\hline & & 960530G01 & P01 & Pingtung Rescue Center & Pingtung Rescue Center \\
\hline & & 26 & 26 & Wuppertal Zoo & German Primate Center \\
\hline & & 501 & 501 & Nuremberg Zoo & German Primate Center \\
\hline & & 502 & 502 & Nuremberg Zoo & German Primate Center \\
\hline & & 2356 & J09 & Dusit Zoo & WRC, Kyoto University \\
\hline & & 2357 & $\mathrm{~J} 10$ & Dusit Zoo & WRC, Kyoto University \\
\hline & & 1981 & $J 26$ & Chiang Mai Zoo & WRC, Kyoto University \\
\hline & & 1982 & $J 27$ & Chiang Mai Zoo & WRC, Kyoto University \\
\hline & & 2845 & $J 28$ & PRI, Kyoto University & WRC, Kyoto University \\
\hline & & 3400 & $J 29$ & PRI, Kyoto University & WRC, Kyoto University \\
\hline & muelleri & 20050386D10 & T16 & Taipei Zoo & Taipei Zoo \\
\hline & & 2520 & $\mathrm{~J} 14$ & Kalimantan Samarinda & WRC, Kyoto University \\
\hline & & 2521 & $\mathrm{~J} 15$ & Kalimantan Samarinda & WRC, Kyoto University \\
\hline & & 1058 & 1058 & Rostock Zoo & German Primate Center \\
\hline & klossii & 1230 & 1230 & Twycross Zoo & German Primate Center \\
\hline & moloch & 2486 & $\mathrm{~J} 12$ & Ragunan Zoo & WRC, Kyoto University \\
\hline & & 2488 & $\mathrm{~J} 13$ & Ragunan Zoo & WRC, Kyoto University \\
\hline & & 2349 & $J 21$ & Ragunan Zoo & WRC, Kyoto University \\
\hline & pileatus & 2360 & J16 & Dusit Zoo & WRC, Kyoto University \\
\hline & & 2361 & J17 & Dusit Zoo & WRC, Kyoto University \\
\hline & & 2362 & $J 22$ & Dusit Zoo & WRC, Kyoto University \\
\hline \multirow[t]{11}{*}{ Symphalangus } & syndactylus & 20050331D10 & T19 & Taipei Zoo & Taipei Zoo \\
\hline & & 20060592D10 & T21 & Taipei Zoo & Taipei Zoo \\
\hline & & 20060392D10 & $\mathrm{T} 22$ & Taipei Zoo & Taipei Zoo \\
\hline & & 223 & 223 & Munich Zoo & German Primate Center \\
\hline & & 659 & 659 & La Vallee des Singes & German Primate Center \\
\hline & & 980 & 980 & Krefeld Zoo & German Primate Center \\
\hline & & 2506 & $\mathrm{~J} 19$ & Ragunan Zoo & WRC, Kyoto University \\
\hline & & 2507 & $J 20$ & Ragunan Zoo & WRC, Kyoto University \\
\hline & & 2512 & $J 32$ & unknown & WRC, Kyoto University \\
\hline & & 1973 & $J 33$ & Padang, Sumatra, Indonesia & WRC, Kyoto University \\
\hline & & 2506 & $J 34$ & unknown & WRC, Kyoto University \\
\hline \multirow[t]{8}{*}{ Nomascus } & leucogenys & 378 & 378 & Twycross Zoo & German Primate Center \\
\hline & & 1005 & 1005 & Duisburg Zoo & German Primate Center \\
\hline & & 1006 & 1006 & Duisburg Zoo & German Primate Center \\
\hline & & 2364 & J04 & Dusit Zoo & WRC, Kyoto University \\
\hline & siki & 1232 & 1232 & London Zoo & German Primate Center \\
\hline & & 1986 & J03 & Chiangmai Zoo & WRC, Kyoto University \\
\hline & gabriellae & Arnold & Arnold & Leipzig Zoo & MPI-EVA \\
\hline & & Falco & Falco & Leipzig Zoo & MPI-EVA \\
\hline
\end{tabular}


Table 2. Cont.

\begin{tabular}{llllll}
\hline & & & & \\
\hline Senus & Species & onwer's ID/Barcode ID & working ID & origin $^{\text {a }}$ & current deposition $^{\text {a }}$ \\
\hline concolor & 1231 & $\mathrm{Nc}$ & Twycross Zoo & German Primate Center \\
\hline
\end{tabular}

aAbbreviations: PRI, Primate Research Institute; WRC, Wildlife Research Center; MPI-EVA, Max-Planck Institute for Evolutionary Anthropology.

doi:10.1371/journal.pone.0014419.t002

\section{Materials and Methods}

\section{DNA samples and whole genome amplification}

A total of 51 high-quality genomic DNA samples representing 11 species were collected (Table 2). All DNA samples used derive from long-term sample collections of the authors and were not acquired specifically for this study. These samples were previously collected from captive gibbons during routine veterinary care. All genomic DNA samples underwent a whole genome amplification (WGA) based upon the multiple displacement amplification procedure using GenomiPhi HY DNA Amplification Kit (GE Healthcare) and were purified by ethanol precipitation following the manufacturer's instructions. The purified WGA products were quantified on a Nanodrop spectrophotometer and used as templates for subsequent long-range PCRs for the amplification of the complete mtgenome.

\section{Two-step multiplex long-range PCR}

We amplified the entire mtgenomes in four large overlapping fragments $(\sim 3.8-5 \mathrm{~kb}$ each) by two-step multiplex long-range PGR using the Expand Long Range dNTPack kit (Roche). In the first multiplex step, the four primer pairs were divided into two sets, A and B (Table 3). Each set contains two pairs of primers (the A set: pairs $1 \times 12$ and $6 \times 9$, the B set: pairs $4 \times 10$ and $5 \times 11$ ) and was used to amplify two non-overlapping fragments differing by $\sim 1 \mathrm{~kb}$. Primers were designed by K. Finstermeier and M. Meyer to function across a wide range of primates and primers modified for use in gibbons were also used, as indicated. This step was performed in a total volume of $50 \mu \mathrm{l}$ containing $100 \mathrm{ng}$ of purified WGA product, $0.3 \mu \mathrm{M}$ each primer, $5 \mathrm{X}$ buffer with $\mathrm{MgCl}_{2}$, $0.5 \mathrm{mM}$ each dNTP and 3.5 unit of the Expand Long Range Enzyme mix under the following cycling conditions: initial denaturation at $92^{\circ} \mathrm{C}$ for $2 \mathrm{~min} ; 9$ cycles of $10 \mathrm{~s}$ at $92^{\circ} \mathrm{C}, 15 \mathrm{~s}$ at $57^{\circ} \mathrm{C}$ and $8 \mathrm{~min}$ at $68^{\circ} \mathrm{C}$; then 21 cycles of $10 \mathrm{~s}$ at $92^{\circ} \mathrm{C}, 15 \mathrm{~s}$ at $57^{\circ} \mathrm{C}$ and $8 \mathrm{~min}$ at $68^{\circ} \mathrm{C}$ increasing by $20 \mathrm{~s}$ each cycle and a final elongation step of $15 \mathrm{~min}$ at $68^{\circ} \mathrm{C}$. The PCR products of the first step were diluted 20 -fold in water and $5 \mu \mathrm{l}$ were used as templates in second step singleplex reactions using the same primer pairs as in the multiplex step. For example, we used diluted PCR products amplified with the set $\mathrm{A}$ as template to amplify the products of $4.1 \mathrm{~kb}$ using the pair $1 \times 12$ (primat_mtl_r and gibbon_mt12-2_f) in the singleplex reaction. The PCR conditions of the second step were the same as in the first, except that the final concentrations of the individual primer pairs was $3 \mu \mathrm{M}$ and annealing temperature was $59^{\circ} \mathrm{C}$. The singleplex PCR products were gel-cut and purified using QIAquick Gel Extraction Kit (Qiagen). The long-range PCR procedure enabled us to reduce the likelihood of amplifying possible nuclear mitochondrial pseudogenes (numts) [27,42,43,44].

\section{Sequencing of mitochondrial genomes}

We used the high-throughput 454 sequencing technology with the parallel tagged sequencing (PTS) approach to sequence the gibbon entire mtgenome. The detailed protocols for preparation are described in Meyer et al. $[45,46]$ and the manufacturer's instructions (GS FLX platform, Roche). In brief, the purified PCR products were pooled by individual in equimolar ratios and sheared. Barcoding adapters with individual-specific tag sequences were ligated to the DNA fragments in each individual pool. A total of 51 unique tags were assigned to the 51 gibbon individuals, respectively. The individual tagged samples were then pooled together in equimolar ratios to produce the sequencing library in which the 454 adaptors were ligated to the tagged fragments. The library was quantified by quantitative PCR and used subsequently in the standard GS FLX sequencing procedure.

The 454 read sequence data were first sorted according to their tag sequences and then classified into the 51 subsets (51 individuals). The reads from each subset were assembled by MIA (Mapping Iterativ Assembler, http://sourceforge.net/projects/ mia-assembler/) to create a consensus contig using the mtgenome

Table 3. Primers used for amplification of the entire mitochondrial genome.

\begin{tabular}{|c|c|c|c|c|}
\hline $\begin{array}{l}\text { Multiplex PCR } \\
\text { sets }\end{array}$ & $\begin{array}{l}\text { Singleplex PCR } \\
\text { pairs }\end{array}$ & primer name & primer sequence $\left(5^{\prime}-3^{\prime}\right)$ & $\begin{array}{l}\text { expected } \\
\text { product size }\end{array}$ \\
\hline \multirow[t]{5}{*}{ A } & $1 \times 12$ & primat_mt1_r & TGTCCTGATCCAACATCGAG & $4.1 \mathrm{~kb}$ \\
\hline & & gibbon_mt12-2_f & CACGARACRGGATCAAACAAY & \\
\hline & $6 \times 9$ & gibbon_mt6-2_r & GGAYCAGGTGACGAAYAGTGC & $5.2 \mathrm{~kb}$ \\
\hline & & gibbon_mt9_f & AGGAAGGAATCGAACCCYC & \\
\hline & & gibbon_mt9-3_f $\mathrm{f}^{b}$ & ACCTTCTTYCCACAACACTTCC & \\
\hline \multirow[t]{4}{*}{ B } & $4 \times 10$ & primat_mt4_f & CCGTGCAAAGGTAGCATAATC & $4.9 \mathrm{~kb}$ \\
\hline & & gibbon_mt10_r & TATGGGGCTGGCTTGAAAC & \\
\hline & $5 \times 11$ & primat_mt5_f & GGCTTTCTCAACTTTTAAAGGATA & $3.8 \mathrm{~kb}$ \\
\hline & & primat_mt11_r & AGAATKYCAGYTTTGGGTRYTG & \\
\hline
\end{tabular}

${ }^{\text {a }}$ The approximate expected lengths of the singleplex PCR products.

${ }^{b}$ The primer gibbon_mt9-3_f was specific for amplification of the $N$. concolor sample.

doi:10.1371/journal.pone.0014419.t003 
of H. lar (GenBank X99256.1) as the reference sequence. The assembled consensus contig of each subset resulted in the mtgenome sequence of each gibbon individual.

\section{Sequence data analyses}

The newly obtained mtgenome sequences were aligned with the H. lar reference using ClustalW 2.0 ([47], http://www.ch.embnet. org/software/ClustalW-XXL.html). The locations of rRNA, tRNA and protein-coding genes were determined by the comparison with the reference and the 37 individual genes were concatenated using DnaSP 5 [48]. To check for the presence of sequences derived from numts, the sequences of protein-coding genes were examined for frameshift or stop mutations and the rRNA sequences were compared with the known siamang rRNA numt (AF420053, [49]). No numt or other anomalous sequences were observed. The control region was excluded from the analyses. The mtgenome sequences of five great apes and one baboon were used as outgroups: Pongo pygmaeus (GenBank NG_001646), Gorilla gorilla (GenBank NC_001645), Pan troglodytes (GenBank NC_001643), Pan paniscus (GenBank NC_001644), Homo sapiens (GenBank AF347008) and Papio hamadryas (GenBank Y18001). The outgoup mtgenomes were processed using BioEdit 7.0.5 [50] to isolate their individual genes and to remove the control regions. The individual genes of outgroups were also concatenated with DnaSP 5. All obtained mtgenome sequences have been deposited in Genbank (Accession numbers HQ622758-HQ622808).

\section{Phylogenetic analyses}

We aligned the concatenated sequences from gibbons and outgroup species using ClustalW. The best-fit nucleotide substitution model was selected by Model-Generator 0.85 [51] and the general time reversible $(\mathrm{GTR})+\mathrm{I}+\Gamma$ model was suggested. We partitioned the 15,484 bp alignment of the concatenated sequences into three schemes comprising (1) $12 \mathrm{~S}$ and 16S rRNA genes combined, rRNA set (2) all 22 tRNA genes combined, tRNA set and (3) all 13 protein-coding genes combined, protein set. The information of alignment length, invariable, variable and informative sites for each scheme is listed in Table 4. We used this partitioned dataset for phylogeny reconstruction applying maximum likelihood (ML), Bayesian and neighbor-joining (NJ) methods as follows. We employed ML in RAxML 7.2.3 ([52,53], http:// phylobench.vital-it.ch/raxml-bb/index.php) with GTR $+\mathrm{I}+\Gamma$ substitution model to each partition. Bootstrap support values were based on 100 replicates. The Bayesian analysis was performed in MrBayes 3.1.2 ([54], http://cbsuapps.tc.cornell.edu/mrbayes. aspx). Four Metropolis-coupled Markov chain Monte Carlo (MCMC) analyses were run twice for 5,000,000 generations and sampled every 100 generations $(\mathrm{mcmcp}$ ngen $=5000000$, nchains $=4$, temp $=0.01$, samplefreq $=100$, burnin $=5000)$. The GTR $+\mathrm{I}+\Gamma$ substitution model was assigned to each partition. The $\mathrm{NJ}$ analysis with the bootstrap test was performed in MEGA 4.0 [55] using pairwise deletion for gaps/missing data. However, due to the unavailability of data partitioning and the GTR model in MEGA, the non-partitioned dataset and the best available TamuraNei model [56] were used for the NJ reconstruction.

\section{Estimation of divergence times}

We estimated the divergence times within the gibbon family using the Bayesian method implemented in the program BEAST
Table 4. Sizes and characteristics of the partitioned datasets.

\begin{tabular}{llll}
\hline Schemes & rRNA & tRNA & Protein \\
\hline Alignment length (bp) & 2,552 & 1,530 & 11,402 \\
No. of invariable sites & 1,135 & 404 & 4,475 \\
No. of variable sites & 488 & 149 & 3,255 \\
No. of informative sites & 326 & 83 & 2,460 \\
\hline
\end{tabular}

doi:10.1371/journal.pone.0014419.t004

1.5.2 ([57], http://cbsuapps.tc.cornell.edu/beast.aspx) with a relaxed molecular clock approach [58]. Three fossil-based calibration points were applied: the split of hominoids-cercopithecoids ( 23 mya \pm 2 mya, $[59,60])$, the separation of Pongo from the Homo/Pan lineage ( $\sim 14$ mya \pm 1 mya, [61]) and the divergence between Homo and Pan ( 6.5 mya \pm 0.5 mya, $[62,63,64])$. Due to the high computational time demand for large datasets in BEAST, we used a sub-dataset of the protein set for analysis instead. In this sub-dataset, 22 gibbons (marked with stars in Figure 2) that are most divergent in their own species lineage and the six outgroup species are included. We partitioned this protein sub-dataset by codon positions (2 partitions: codon positions [1+2], 3) and unlinked the substitution model, rate heterogeneity and base frequencies across them. The tree topology obtained from abovementioned phylogenetic analyses and the GTR $+\mathrm{I}+\Gamma$ substitution model were implemented in BEAST with the following settings: an uncorrelated lognormal relaxed clock model, Yule speciation process in tree prior, and 50,000,000 generations of MCMC steps sampled every 5000 generations. Two independent BEAST runs were carried out and the log output files were combined using LogCombiner 1.5.3 [57]. The effective sample size (ESS) values (greater than 380) were adequate for all parameters. Convergence was assessed in Tracer 1.5 (http:// treebioedacuk/software/tracer/) and the first 1000 samples (5,000,000 generations) were excluded as burn-in. The chronological tree files were analyzed and visualized using TreeAnnotator 1.5.3 [57] and FigTree 1.3.1 (http://tree.bio.ed.ac.uk/software/ figtree/).

\section{Acknowledgments}

We thank K. Finstermeier for providing the sequences of primate primers and helping with the gibbon-specific primer design. We are grateful to the following colleagues, zoos and institutions for providing valuable gibbon materials: the late Professor Osamu Takenaka of Kyoto University, Dr. Bambang Suryobroto of Bogor Agricultural University, Dr. Suchinda Malaivijitnond of Chulalongkorn University, Bristol Zoo, Duisburg Zoo, Krefeld Zoo, La Vallee des Singes, Leipzig Zoo, London Zoo, Mulhouse Zoo, Munich Zoo, Nuremberg Zoo, Rostock Zoo, Twycross Zoo, Wuppertal Zoo, Chiang Mai Zoo, Dusit Zoo, Ragunan Zoo, Taipei Zoo, Primate Reseearch Institute of Kyoto University, Japan Monkey Centre and Pingtung Rescue Center of Endangered Wild Animals.

\section{Author Contributions}

Conceived and designed the experiments: LV. Performed the experiments: YCC. Analyzed the data: YCC CR. Contributed reagents/materials/ analysis tools: CR MIM EI CCS KJCP LV. Wrote the paper: YCC CR LV.

2. Brandon-Jones D, Eudey AA, Geissmann T, Groves CP, Melnick DJ, et al. (2004) Asian primate classification. Int J Primatol 25: 97-164. 
3. Mootnick A, Groves C (2005) A new generic name for the hoolock gibbon (Hylobatidae). Int J Primatol 26: 971-976.

4. Thinh VN, Mootnick AR, Geissmann T, Li M, Ziegler T, et al. (2010) Mitochondrial evidence for multiple radiations in the evolutionary history of small apes. BMC Evol Biol 10: 74.

5. IUCN Red List 2010. http://wwwiucnredlistorg/.

6. Roos C, Geissmann T (2001) Molecular phylogeny of the major hylobatid divisions. Mol Phylogenet Evol 19: 486-494.

7. Müller S, Hollatz M, Wienberg J (2003) Chromosomal phylogeny and evolution of gibbons (Hylobatidae). Hum Genet 113: 493-501.

8. Takacs Z, Morales JC, Geissmann T, Melnick DJ (2005) A complete specieslevel phylogeny of the Hylobatidae based on mitochondrial ND3-ND4 gene sequences. Mol Phylogenet Evol 36: 456-467.

9. Monda K, Simmons RE, Kressirer P, Su B, Woodruff DS (2007) Mitochondrial DNA hypervariable region- 1 sequence variation and phylogeny of the concolor gibbons, Nomascus. Am J Primatol 69: 1285-1306.

10. Whittaker DJ, Morales JC, Melnick DJ (2007) Resolution of the Hylobates phylogeny: congruence of mitochondrial D-loop sequences with molecular, behavioral, and morphological data sets. Mol Phylogenet Evol 45: 620-628.

11. Roos C, Vu Ngoc Thanh, Walter L, Nadler T (2007) Molecular systematics of Indochinese primates. Vietn J Primatol 1: 41-53.

12. Garza JC, Woodruff DS (1992) A phylogenetic study of the gibbons (Hylobates) using DNA obtained noninvasively from hair. Mol Phylogenet Evol 1: 202-210.

13. Hayashi S, Hayasaka K, Takenaka O, Horai S (1995) Molecular phylogeny of gibbons inferred from mitochondrial-DNA sequences - preliminary-report. J Mol Evol 41: 359-365.

14. Raaum RL, Sterner KN, Noviello CM, Stewart CB, Disotell TR (2005) Catarrhine primate divergence dates estimated from complete mitochondrial genomes: concordance with fossil and nuclear DNA evidence. J Hum Evol 48: $237-257$.

15. Matsudaira K, Ishida T (2010) Phylogenetic relationships and divergence dates of the whole mitochondrial genome sequences among three gibbon genera. Mol Phylogenet Evol 55: 454-459.

16. Matsui A, Rakotondraparany F, Munechika I, Hasegawa M, Horai S (2009) Molecular phylogeny and evolution of prosimians based on complete sequences of mitochondrial DNAs. Gene 441: 53-66.

17. Schrago CG, Russo CAM (2003) Timing the origin of New World monkeys. Mol Biol Evol 20: 1620-1625.

18. Chatterjee HJ (2006) Phylogeny and biogeography of gibbons: a dispersalvicariance analysis. Int J Primatol 27: 699-712

19. Ingman M, Kaessmann H, Paabo S, Gyllensten U (2000) Mitochondrial genome variation and the origin of modern humans. Nature 408: 708-713.

20. Miya M, Kawaguchi A, Nishida M (2001) Mitogenomic exploration of higher teleostean phylogenies: a case study for moderate-scale evolutionary genomics with 38 newly determined complete mitochondrial DNA sequences. Mol Biol Evol 18: 1993-2009.

21. Delisle I, Strobeck C (2005) A phylogeny of the Caniformia (order Carnivora) based on 12 complete protein-coding mitochondrial genes. Mol Phylogenet Evol 37: 192-201.

22. Yu L, Li YW, Ryder OA, Zhang YP (2007) Analysis of complete mitochondrial genome sequences increases phylogenetic resolution of bears (Ursidae), a mammalian family that experienced rapid speciation. BMC Evol Biol 7: 198.

23. Krause J, Unger T, Nocon A, Malaspinas AS, Kolokotronis SO, et al. (2008) Mitochondrial genomes reveal an explosive radiation of extinct and extant bears near the Miocene-Pliocene boundary. BMC Evol Biol 8: 220.

24. Morin PA, Archer FI, Foote AD, Vilstrup J, Allen EE, et al. (2010) Complete mitochondrial genome phylogeographic analysis of killer whales (Orcinus orca) indicates multiple species. Genome Res 20: 908-916.

25. Zhang P, Papenfuss TJ, Wake MH, Qu L, Wake DB (2008) Phylogeny and biogeography of the family Salamandridae (Amphibia: Caudata) inferred from complete mitochondrial genomes. Mol Phylogenet Evol 49: 586-597.

26. Rohland N, Malaspinas AS, Pollack JL, Slatkin M, Matheus P, et al. (2007) Proboscidean mitogenomics: chronology and mode of elephant evolution using mastodon as outgroup. PLoS Biol 5: 1663-1671.

27. Thalmann O, Hebler J, Poinar HN, Paabo S, Vigilant L (2004) Unreliable mtDNA data due to nuclear insertions: a cautionary tale from analysis of humans and other great apes. Mol Ecol 13: 321-335.

28. Hall LM, Jones DS, Wood BA (1998) Evolution of the gibbon subgenera inferred from cytochrome b DNA sequence data. Mol Phylogenet Evol 10: 281-286.

29. Arnason U, Bodin K, Gullberg A, Ledje C, Mouchaty S (1995) A molecular view of pinniped relationships with particular emphasis on the true seals. J Mol Evol 40: 78-85.

30. Geissmann $\mathrm{T}$ (1995) Gibbon systematics and species identification. Int Zoo News 42: 65-77.

31. Johnson WE, Eizirik E, Pecon-Slattery J, Murphy WJ, Antunes A, et al. (2006) The late Miocene radiation of modern Felidae: a genetic assessment. Science 311: 73-77.

32. Gilbert C, Ropiquet A, Hassanin A (2006) Mitochondrial and nuclear phylogenies of Cervidae (Mammalia, Ruminantia): systematics, morphology, and biogeography. Mol Phylogenet Evol 40: 101-117.

33. Fulton TL, Strobeck C (2010) Multiple fossil calibrations, nuclear loci and mitochondrial genomes provide new insight into biogeography and divergence timing for true seals (Phocidae, Pinnipedia). J Biogeography 37: 814-829.
34. Cerling TE, Ehleringer JR, Harris JM (1998) Carbon dioxide starvation, the development of C4 ecosystems, and mammalian evolution. Philos Trans R Soc Lond B Biol Sci 353: 159-170; discussion 170-151.

35. Fortelius M, Eronen J, Liu LP, Pushkina D, Tesakov A, et al. (2006) Late Miocene and Pliocene large land mammals and climatic changes in Eurasia. Palaeogeogr Palaeoclimatol Palaeoecol 238: 219-227.

36. Gladenkov AY, Oleinik AE, Marincovich L, Barinov KB (2002) A refined age for the earliest opening of Bering Strait. Palaeogeogr Palaeoclimatol Palaeoecol 183: $321-328$.

37. Mittermeier R, Gil P, Hoffman M, Pilgrim J, Brooks T, et al. (2005) Hotspots revisited: Earth's biologically richest and most endangered terrestrial ecoregions. Washington: Conservation International

38. Quek SP, Davies SJ, Ashton PS, Itino T, Pierce NE (2007) The geography of diversification in mutualistic ants: a gene's-eye view into the Neogene history of Sundaland rain forests. Mol Ecol 16: 2045-2062.

39. Outlaw DC, Voelker G (2008) Pliocene climatic change in insular Southeast Asia as an engine of diversification in Ficedula flycatchers. J Biogeography 35: 739-752.

40. den Tex RJ, Thorington R, Maldonado JE, Leonard JA (2010) Speciation dynamics in the SE Asian tropics: putting a time perspective on the phylogeny and biogeography of Sundaland tree squirrels, Sundasciurus. Mol Phylogenet Evol 55: 711-720.

41. Woodruff DS, Turner LM (2009) The Indochinese-Sundaic zoogeographic transition: a description and analysis of terrestrial mammal species distributions. J Biogeography 36: 803-821.

42. Bensasson D, Zhang DX, Hartl DL, Hewitt GM (2001) Mitochondrial pseudogenes: evolution's misplaced witnesses. Trends Ecol Evol 16: 314-321.

43. Akanuma J, Muraki K, Komaki H, Nonaka I, Goto Y (2000) Two pathogenic point mutations exist in the authentic mitochondrial genome, not in the nuclear pseudogene. J Hum Genet 45: 337-341.

44. Fenn JD, Song H, Cameron SL, Whiting MF (2008) A preliminary mitochondrial genome phylogeny of Orthoptera (Insecta) and approaches to maximizing phylogenetic signal found within mitochondrial genome data. Mol Phylogenet Evol 49: 59-68.

45. Meyer M, Stenzel U, Myles S, Prufer K, Hofreiter M (2007) Targeted highthroughput sequencing of tagged nucleic acid samples. Nucleic Acids Res 35.

46. Meyer M, Stenzel U, Hofreiter M (2008) Parallel tagged sequencing on the 454 platform. Nat Protoc 3: 267-278.

47. Larkin MA, Blackshields G, Brown NP, Chenna R, McGettigan PA, et al. (2007) Clustal W and Clustal X version 2.0. Bioinformatics 23: 2947-2948.

48. Librado P, Rozas J (2009) DnaSP v5: a software for comprehensive analysis of DNA polymorphism data. Bioinformatics 25: 1451-1452.

49. Schmitz J, Piskurek O, Zischler H (2005) Forty million years of independent evolution: a mitochondrial gene and its corresponding nuclear pseudogene in primates. J Mol Evol 61: 1-11.

50. Hall TA (1999) A user-friendly biological sequence alignment editor and analysis program for Windows 95/98/NT. Nucleic Acids Symp Ser 41: 95-98.

51. Keane TM, Creevey CJ, Pentony MM, Naughton TJ, McLnerney JO (2006) Assessment of methods for amino acid matrix selection and their use on empirical data shows that ad hoc assumptions for choice of matrix are not justified. BMC Evol Biol 6: 29.

52. Stamatakis A (2006) RAxML-VI-HPC: maximum likelihood-based phylogenetic analyses with thousands of taxa and mixed models. Bioinformatics 22: $2688-2690$

53. Stamatakis A, Hoover P, Rougemont J (2008) A rapid bootstrap algorithm for the RAxML Web servers. Syst Biol 57: 758-771.

54. Ronquist F, Huelsenbeck JP (2003) MrBayes 3: Bayesian phylogenetic inference under mixed models. Bioinformatics 19: 1572-1574.

55. Tamura K, DudleyJ, Nei M, Kumar S (2007) MEGA4: Molecular Evolutionary Genetics Analysis (MEGA) software version 4.0. Mol Biol Evol 24: 1596-1599.

56. Tamura K, Nei M (1993) Estimation of the number of nucleotide substitutions in the control region of mitochondrial DNA in humans and chimpanzees. Mol Biol Evol 10: 512-526.

57. Drummond AJ, Rambaut A (2007) BEAST: Bayesian evolutionary analysis by sampling trees. BMC Evol Biol 7: 214

58. Drummond AJ, Ho SY, Phillips MJ, Rambaut A (2006) Relaxed phylogenetics and dating with confidence. PLoS Biol 4: e88.

59. Benefit BR, McCrossin ML (2002) The Victoriapithecidae, Cercopithecoidea. In: C HW, ed. The primate fossil record. Cambridge: Cambridge University Press. pp 241-253.

60. Young NM, MacLatchy L (2004) The phylogenetic position of Morotopithecus. J Hum Evol 46: 163-184.

61. Kelley J (2002) The hominoid radiation in Asia. In: Hartwig WC, ed. The primate fossil record. Cambridge: Cambridge University Press. pp 369-384.

62. Vignaud P, Duringer P, Mackaye HT, Likius A, Blondel C, et al. (2002) Geology and palaeontology of the Upper Miocene Toros-Menalla hominid locality, Chad. Nature 418: 152-155.

63. Brunet M, Guy F, Pilbeam D, Lieberman DE, Likius A, et al. (2005) New material of the earliest hominid from the Upper Miocene of Chad. Nature 434: $752-755$.

64. Lebatard AE, Bourles DL, Duringer P, Jolivet M, Braucher R, et al. (2008) Cosmogenic nuclide dating of Sahelanthropus tchadensis and Australopithecus bahrelghazali: Mio-Pliocene hominids from Chad. Proc Natl Acad Sci U S A 105: 3226-3231. 